\title{
CONSIDERACIONES GENERALES SOBRE EL VOCABULARIO JURÍDICO GRIEGO
}

José María Lucas

U.N.E.D.

\section{Planteamiento de la cuestión}

1.1. Frente al criterio, ampliamente sostenido, de que los griegos no dispusieron de un vocabulario técnico ${ }^{1}$ en el campo de sus relaciones jurídicas, ya públicas ya privadas, querría con estas líneas romper alguna lanza a favor de la tesis contraria: creo que puede detectarse perfectamente una clara tendencia en la lengua griega a ir consolidando un vocabulario jurídico en el mismo sentido que posteriormente sucederá en el Derecho Romano o en los sistemas legales de nuestros días, aunque, eso sí, en el caso del Derecho griego todavía es perceptible toda una serie de vacilaciones, reflejo de que en Grecia no se llegó realmente a constituir un corpus jurídico tan acabado y homogéneo como en sus sucesores occidentales.

1.2. Y, a mi juicio, la cuestión no tiene sólo una transcendencia meramente lingüística, por lo que supone de un mayor conocimiento semántico del griego; sino que la oportuna consideración de los diversos mecanismos léxicos en la esfera jurídica puede ser de importancia básica a la hora de analizar una institución legal determinada, dentro ya del campo del Derecho propiamente dicho. Por ejemplo, una reflexión de este tipo pienso que habría sido muy útil en la polémica surgida hace cinco años entre Rhodes $^{2}$ y Hansen ${ }^{3}$ a colación de la "acción pública» conocida con el término de

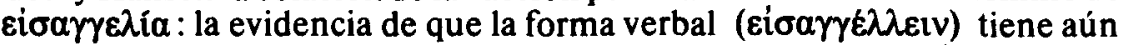

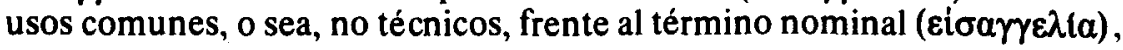

1 Prefiero utilizar el térmmino «vocabulario» y no «lenguaje» porque en esta parcela de la lengua se trata siempre de hechos simplemente de léxico, sin unas características especiales en el campo fonológico, o sintáctico, o incluso en la propia morfología, puesto que, como más abajo veremos, este tipo de terminología técnica no utiliza, tanto en la derivación como en la composición, unos morfemas especiales, sino que se atiene a los procedimientos de la lengua, sin especialización de ninguna clase.

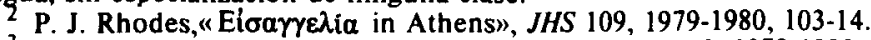

3 M. H. Hansen, «Eisangelia in Athen: a reply», JHS 109, 1979-1980, 89-95. 
que sólo aparece empleado como tecnicismo jurídico, pone en evidencia que la aparición del primero no presupone en un texto dado la existencia de este proceso concreto. Dicho de otra manera más general, hay términos que, a juzgar al menos por los textos conservados, tienen un empleo exclusivamente jurídico $y$, en este sentido, su aparición supone un valor preciso del contexto legal en que aparecen; pero, por el contrario, en otras ocasiones nos encontramos con vocablos de sentido alternante entre el uso común y un valor técnico, en cuyo caso será preciso tener una mayor cautela a la hora de interpretar el pasaje. Y estas consideraciones que para un lingüista son hechos elementales de vocabulario, con mucha frecuencia son dejadas de lado por los historiadores, con el riesgo consiguiente de interpretación. Aunque también no es menos cierto que en ocasiones los primeros no van más allá de meras reflexiones lingüísticas, expuestas a errores interpretativos dado su desconocimiento de los hechos de realia. Tal vez, pues, estemos ante un nuevo caso de necesaria colaboración entre ambos campos.

\section{Situación PROBlemática}

2.1. Pues bien, si se admite la utilidad de ese añalisis pormenorizado del vocabulario jurídico, surge inmediatamente la necesidad de perfilar los grandes rasgos que caracterizan este tipo de léxico, los diversos procedimientos de creación y evolución que ha experimentado, a la luz de todo lo cual podamos después enfrentarnos con rigor y seguridad a los casos concretos. Y es evidente que el camino a recorrer es doble: primero habrá que ir de las palabras individuales a las categorías y conceptos globalizadores, para a continuación estar en disposición de aplicar esos resultados anteriores a cada texto en particular.

Pero, por lo que yo sé, hay una carencia total de estudios globales de este vocabulario en el sentido que arriba apunto. Por supuesto que existe gran abundancia de trabajos parciales de términos o conceptos jurídicos, pero casi siempre desde la perspectiva del fondo técnico legal, sin recapacitar que la lengua que expresa tales relaciones es una realidad mucho más compleja, puesto que al tiempo que es reflejo de las diversas estructuras institucionales, no es menos cierto que es algo más amplio, vehículo de comunicación general entre los miembros de esa misma sociedad, y que, para bien o para mal, es el único puente de unión entre el individuo y los referentes del entorno.

Y si desde el punto de vista semántico-lexicológico la deficiencia en este momento es grande, la ayuda desde el terreno lexicográfico tampoco es importante. En los grandes diccionarios generales, por ejemplo el LiddellScott-Jones (L-S-J), hay un desinterés generalizado por cuestiones de este tipo $^{4}$. Y sin extenderme demasiado en este punto, que me llevaria muy lejos

${ }^{4}$ Este es un nuevo aspecto en el que puede apreciarse la distancia entre la obra lexicográ- 
de lo que persigo en estas páginas, yo diría que debe haber, al menos, dos consideraciones básicas a la hora de introducir este tipo de vocabulario técnico en una obra lexicográfica general: 1) es preciso marcar de forma rotunda el corte entre un significado de la lengua común y el sentido específico de un término, cosa que con frecuencia no se hace, a no ser, claro está, en palabras clásicas - para tales casos debería generalizarse la apertura de un apartado nuevo con número propio, dentro del cual, además, habría que ir clasificando las variaciones temporales o geográficas que conlleva este tipo de vocabulario, aspecto este sobre el que volveré más abajo-; y 2) hay que ser extricto con el plano cronológico, y dejar bien en claro si un vocablo, a juzgar al menos por la documentación de que disponemos, tiene en principio un empleo técnico del que evolucionará hasta convertirse en expresión de la lengua común, o si, por el contrario, el proceso de su evolución significativa es en el sentido inverso, del uso común a la esfera especializada.

También es escasa la ayuda que nos brindan los trabajos lexicográficos más particulares. No existe, a pesar de su evidente ayuda, un diccionario de terminología jurídica griega ${ }^{5}$. Disponemos, a lo sumo, de una serie de İndices de los oradores áticos ${ }^{6} \mathrm{y}$ de algunas colecciones de inscripciones, pero la sola mención del concepto de Índice ya deja a las claras la reducida utilidad para una visión global de nuestro vocabulario. A todo esto, por supuesto, habría que añadir la parte que ocupa este campo del Derecho griego en los diversos diccionarios enciclopédicos de la Antigüedad: la $R E$, el Daremberg-Saglio, Der kleine Pauly, Artemis, The Oxford classical Dictionary, etc.; o aquellas obras de orientación juridica que recogen también la aportación de Grecia al área de la jurisprudencia, como el Digesto Italiano (49 vols., 18841921), o sus dos posteriores ediciones, muy diversas entre sí, del Nuovo Digesto Italiano (13 vols., 1937-1940, con la colaboración central de Paoli en el terreno que nos interesa), o del Novissimo Digesto Italiano (20 vols. +5 apéndices por ahora, 1957-1984, con participación de Paoli, Biscardi, Eva Cantarella, Lepri y otros) ${ }^{7}$. Pero, a mi juicio, este tipo de obras apenas suponen ayuda alguna desde la perspectiva del estudio del vocabulario en $\mathbf{s i}^{8}$.

fica inglesa y el Diccionario Griego-Español (DGE) que en Madrid está llevando a cabo un equipo de investigadores bajo la dirección del Prof. Adrados.

La propia Antigüedad ya sintió la necesidad de recopilar el vocabulario propio de los oradores, dentro del cual lógicamente ocupan un lugar destacado los términos jurídicos. Se nos ha conservado el Léxico de los diez oradores de Harpocración, que vivió en el s. I/II d. C. Pero tenemos noticia de otras obras semejantes: por ejemplo, en Focio, Biblioteca, 150 se nos mencionan tres autores que compusieron sendas obras lexicográficas sobre esta área: Juliano -homónimo del emperador-, Filóstrato de Tiro y Diodoro. Los tres son personajes de segunda fila, pero su número deja a las claras el interés que ofrecía este tipo de obra.

6 No he podido consultar la obra de K. Schodorf, Beiträge zur genaueren Kenntnis der attischen Gerichtssprache aus den 10 Rednern, Würzburg, 1904.

7 También hay información al respecto en la Enciclopedia italiana di scienza, lettere ed arti (36 vols., 1929-1939).

8 Existe alguna relación parcial de terminología jurídica e institucional, como el peque- 
2.2. Además, en el caso de este tipo de vocabulario la situación se complica con el problema de la «unidad del Derecho griego». 6Existió en la Grecia antigua una única norma jurídica, aunque con pequeñas variaciones según las póleis, o realmente hay que hablar de instituciones y comportamientos legales independientes en las diversas ciudades-estado helénicas? El primer intento de dar solución al problema, como es sabido, se remonta a Mitteis, que postuló una "afinidad racial» aunque con «pequeños matices diferentes» a finales del siglo pasado'. Pero el debate, cual otra cuestión homérica, se ha mantenido hasta nuestros días con apariciones periódicas ${ }^{10}$.

En ocasiones se ha recurrido al paralelismo con la fragmentación dialectal del griego como hecho cultural semejante, $y$ en esta dirección creo que podríamos tal vez establecer el trinomio de arcaísmos, elecciones e innovaciones de que hace treinta años hablaba Adrados al abordar el problema de los dialectos como un reflejo más de la migración indoeuropea en suelo helénico ${ }^{11}$ : al igual que hay que hablar de dialectos o grupos dialectales verdaderamente consolidados por obra de una serie de innovaciones lingüisticas peculiares a cada uno de ellos frente a los demás, en igual medida podríamos admitir la existencia de particularismos legales que han dado lugar en las diferentes póleis a corpora jurídicos específicos. Pero ello no es obstáculo para que pueda mantenerse elementos comunes, restos de la antigua comunidad, así como otros, variables entre áreas más amplias de población, que no serían más que comportamientos alternativos que en un momento dado aún no había adquirido la categoría de normas legales de obligado cumplimiento en una colectividad dada, sino que no eran más que variantes consuetudinarias que posteriormente, en un sentido u otro, fueron adquiriendo el rango de institución jurídica según la elección de cada grupo social, dándose la posibilidad de que puedan coincidir algunos entre sí frente a otros que adoptaron una posibilidad alternativa.

Ahora bien, por lo que atañe a la cuestión del vocabulario, no es necesario insistir mucho en la complicación que supone esta diversidad jurídica en tre las distintas póleis en un momento sincrónico del proceso: un mismo término puede tener en una colectividad dada un empleo técnico concreto, mientras que en otra simplemente funcionar como un vocablo de la lengua común; o también, es perfectamente posible que la misma palabra recubra una realidad institucional bastante diferente en dos comunidades contemporáneas.

ño léxico de términos técnicos en el último volumen de los discursos privados de Demóstenes de la colección Budé, a cargo de $\mathbf{R}$. Weil, que es un buen auxiliar en los primeros momentos.

${ }_{9}$ L. Mitteis, Reichsrecht und Volksrecht in den östlichen Provinzen des Römischen Kaiserreichs, Leipzig, 1891 (reimpr., Hildesheim, 1963).

${ }^{10}$ Entre las últimas menciones cf. A. Biscardi Diritto greco antica, Giuffrè editore, Varese, 1982, pp. 7-9, que en nota 9 de p. 8 hace un breve resumen de la diatriba con las indicaciones bibliográficas pertinentes.

11 F. R. Adrados, La dialectologia grieg a como fuente para el estudio de las migraciones indoeuropeas en Grecia, Salamanca, 1952. 
2.3. Pero los problemas no paran aquí. Como sucede con el vocabulario de otros campos de la ciencia o técnicos en general, por ejemplo el de la Medicina griega ${ }^{12}$, en los textos que se nos han transmitido hay una evolución semántica extraverbal en este tipo de léxico, puesto que es evidente que un mismo término recubre realidades institucionales diversas en momentos distintos del devenir histórico de una misma colectividad. Y este hecho nos fuerza a tener que prestar en todo momento una atención rigurosa al plano diacrónico, a la par que al geográfico aludido en el punto anterior.

\section{CREACIÓN DE UN VOCABULARIO JURIDICO EN GRECIA}

3.1. Vocabulario común con valor juridico. Tras el análisis individual y pormenorizado del material léxico llegamos a establecer esta primera categoría, en la que vemos cómo una serie de términos, que originalmente tienen un sentido de la lengua común, en un momento dado son utilizados para expresar un contenido preciso del campo del Derecho.

En este apartado hay que incluir, claro está, todas esas palabras que, a partir de un significado más concreto, han terminado por alcanzar el valor de realidades abstractas, en este caso en relación con el mundo de las estructuras legales de la comunidad helénica ${ }^{13}$. Me estoy refiriendo, efectivamente,

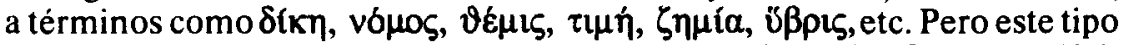
de material léxico ha gozado siempre de la atención de los filólogos clási$\cos ^{14}$, por lo que no voy a entrar aquí en su consideración, sino que me referiré preferentemente al vocabulario más particular $y$, por otra parte, más especifico del Derecho griego.

El otro extremo de esta área léxica lo constituyen una serie de términos cuyo valor no es rigurosamente jurídico, sino que fluctúa entre un uso común y su empleo técnico, puesto que realmente contiene siempre unos componentes específicamente legales. Es algo que sucede también en las demás lenguas, por ejemplo en castellano con palabras como «casarse» o lexías complejas como «llevar a juicio». Ahora bien, aunque efectivamente es discutible la oportunidad o no de calificar esta clase de vocabulario como técnicamente jurídico, no es menos cierto que en ocasiones podemos descubrir en él una evolución desde un significado eminentemente neutro a

12 Cf. J. Alsina, «Sobre los origenes de la lengua médica griega», BIEH 9, 1975, 69-70.

13 Es el mismo fenómeno semántico acaecido a una gran parte de los términos abstractos. Cf., por ejemplo, el proceso de Bíos en E. Gangutia, Vida/muerte de Homero a Platón, Madrid, 1977.

4 Dejando a un lado el siglo XIX, cf. ya desde los primeros decenios del XX obras como $R$. Hirzel, Themis, Dike und verwandtes. Beitrag zur Geschichte der Rechtsidee bei den Griechen, Leipzig, 19/7; o L. Gernet, Recherches sur le developpement de la pensée juridique et morale en Grèce. París. 1917. 
un valor con una realidad institucional próxima al campo del Derecho, lo cual es reflejo de su situación ambigua.

Observemos de cerca, por ejemplo, la evolución semántica de ouvol$\kappa \hat{\varepsilon} \omega$. El término está claramente compuesto del prefijo $\sigma u v$ - y del verbo oi$\kappa \varepsilon \omega$, y su significado más elemental es lógicamente "vivir con", "convivir", como podemos ver en múltiples textos: en Soph. Tr. 545-546 oimos a Deyanira lamentarse de su situación de tener que convivir próximamente con Yola, una vez que Licas ha dado a conocer, primero, la próxima llegada de Heracles y, posteriormente, explicado la auténtica realidad de las circunstancias

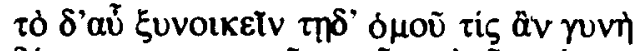

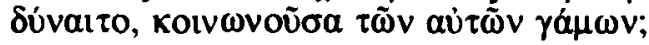

Y es claro que el paso semántico hasta llegar a significar "vivir con alguien en unión matrimonial», "estar casado» fue fácil. Pero en este caso disponemos de una serie de textos que nos ilustran el camino: en Eur. Med. 241-242 la heroína, en su conocido alegato feminista, alude a la posibilidad de una convivencia matrimonial armónica,

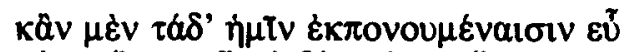

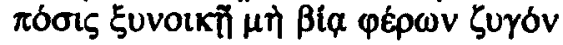

y en este texto podemos percibir que estamos a medio camino del recorrido: el sentido central del término está aludiendo al concepto de «convivir», aunque no es menos cierto que tanto el contexto como el empleo del voca-

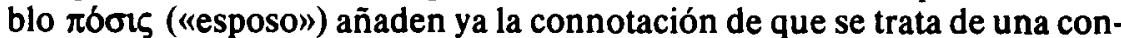
vivencia dentro del matrimonio. Un paso adelante en la evolución lo encontramos en Eur. Andr. 17-18, donde la en otro tiempo feliz esposa de Héctor y ahora, por los ingratos cambios del destino, esclava del joven Neoptólemo hace recuento de su accidentada existencia, que la ha llevado a habitar los mismos campos donde antaño Tetis vivía unida en matrimonio a Peleo, y en este caso pienso que el énfasis no se centra tanto en el concepto de «convivencia» sino en el de pasar la vida en unión matrimonial, circunstancia esta última que no viene ya precisada ni por el contexto del pasaje ni por elemento alguno de la frase, sino por el fondo mitológico conocido de todos. Y el punto final de esta andadura, donde ya la significación no es más que la de "estar casado", lo encontramos en Hdt. I,93.4, donde se nos narra la costumbre de las mujeres lidias de ejercer la prostitución como medio de ir reuniendo la dote matrimonial, práctica ésta en la que se mantienen hasta que se casan: 


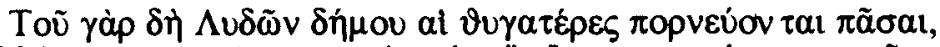

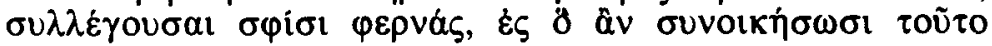

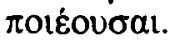

y que en este texto de Heródoto estamos ante un ejemplo semánticamente puro nos lo demuestra su empleo sintáctico absoluto, en el que no hay determinantes sintácticos ni contextuales ni extralingüisticos que configuren el significado de nuestro término.

Hasta aquí he venido hablando de las dos posibles zonas limitrofes de este campo lexical del griego antiguo: los términos conceptuales y abstractos, que tienen su incidencia en otras áreas del pensamiento helénico, $y$, en el otro extremo, esa serie de palabras a caballo entre su u so común y un cierto tecnicismo, dado que contienen siempre unos componentes legales. $Y$ ello, claro está, sin perder de vista que estamos aún en el apartado inicial de Vocabulario común con valor juridico. Pues bien, entre uno y otro límite nos encontramos con un abundante material léxico que, paralelamente a su valor en la lengua común y por la presión de contextos específicamente juridicos, adquiere una significación plenamente técnica y, por lo tanto, muy alejada de su sentido en el nivel general de la lengua. Ahora bien, el análisis pormenorizado de dicho material permite a mi juicio establecer una triple subdivisión, en función de la relevancia que haya adquirido el nuevo significado ${ }^{15}$.

3.1.1. Uso alternativo. Se trata de aquel vocabulario que mantiene a todo lo largo de la tradición literaria del griego antiguo su doble uso: en la lengua común, con sus diversas acepciones, y en el campo de la terminología jurídica; $y$ esta permanencia de valores se da manera equilibrada, sin detrimento de los valores tradicionales. Los ejemplos de esta coincidencia cronológica son innumerables y no voy, por ello, a detenerme a ejemplificarla. Sólo convendría añadir que es el apartado mayoritario, lo que habla del estado de nacimiento incipiente de este tipo de vocabulario.

Al lado de esta coincidencia cronológica se da también, como sucede igualmente en nuestras lenguas modernas, coincidencia dentro de un mismo autor, que emplea el mismo término unas veces en un sentido de la lengua común y otras con valor jurídico. Por poner un ejemplo entre mil podríamos ver cómo el vocablo $\pi p o ́ \sigma 0 \delta$ os, que en su acepción más general equivale a "entrada», adopta el sentido de «ingreso» en un contexto crematístico como Aesch. III, 173:

is La gradación de empleos que a continuación establezco, se basa en el Liddell-ScottJones, lo que supone evidentemente una limitación ya que, como es sabido, se trata de una obra lexicográfica selectiva; pero la carencia de un Thesaurus de la lengua griega nos impone tal restricción. No obstante, suele ser admitida por todos la oportunidad en la selección de los materiales del L-S-J, lo que afianza las diversas clasificaciones aquí propuestas. 


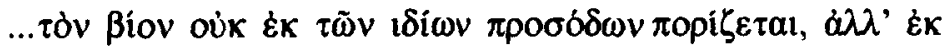

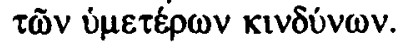

con un empleo metafórico sencillo. Pero, a la par, en Aesch. II, 59, dentro ya del campo jurídico, equivale a "audiencia", "acción de hacerse uno oír en sus reclamaciones ante un órgano legal», significado este claramente ya más lejano de aquel valor local de "entrada", "acceso":

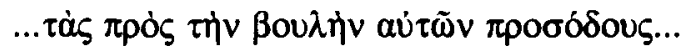

De otro lado, este tipo de léxico se rige por los mismos procedimientos que el resto del vocabulario griego. Por ejemplo: sabemos que los adjetivos en - $\tau o ́ c ̧$ pueden presentar alternativamente un significado pasivo o activo ${ }^{16}$, y así vemos cómo el término $\pi$ oın $\tau^{\prime} \varsigma^{17}$ se utiliza tanto para la realidad del hijo "adoptado» como para la del padre "adoptante»" ${ }^{18}$.

Finalmente, en la mayoría de los casos es fácilmente perceptible el proceso sintáctico-semántico que ha llevado a la creación de estos valores jurídicos en palabras de la lengua común. Pongamos un ejemplo: el fenómeno

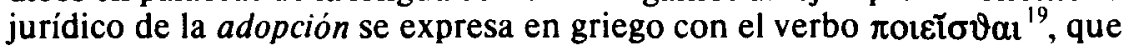
lleva un acusativo de la persona adoptada y, en ocasiones, el predicativo vióv: (D. XLIV, 46)

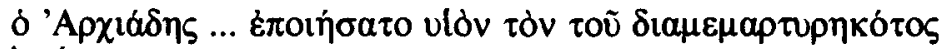
vovi $\pi \dot{a} \pi \pi$ ov.

Pues bien, en estas circunstancias es perfectamente admisible sostener que la construcción con tal predicativo es el punto intermedio en la evolución semántica, puesto que en textos de este tipo es todavia rastreable una interpretación como "hacer a uno hijo", donde vemos que la significación común de «hacer» se mantiene aún con una cierta vigencia; cosa que no sucede en aquellos textos en que falta dicho predicativo, en cuyo caso es el contexto general el que impone el sentido de "adoptar", como sucede, por ejemplo, en Is. II, 1:

16 Cf. P. Chantraine, La formation des noms en grec ancien. París, 1933, pp. 306 y ss.

17 También se usa $\varepsilon$ loroı $t \delta \delta$, ambos con referencia al padre adoptante, mientras que

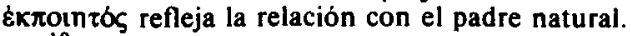

18 Para esta institución legal se utiliza también el término $\zeta \varepsilon \tau o ́ s$, que también adopta a su vez la doble función pasiva y activa aludida.

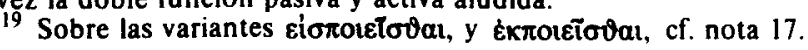




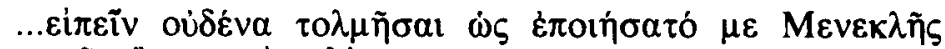

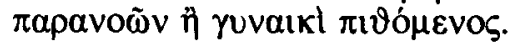

3.1.2. Uso preferente. Frente al empleo alternativo; o sea, equilibrado, del apartado anterior, nos encontramos con un grupo de términos en los que es perceptible ya una clara tendencia al predominio del nuevo valor jurídico, en detrimento del sentido correspondiente a la lengua común. O dicho de otra manera, tras la aparición del significado técnico hay un claro abandono del uso común de dicho vocablo, perfilándose una determinada especialización.

Por poner un ejemplo, podríamos hablar de ě $\varphi \varepsilon \sigma \iota \zeta$ como término adoptado para el proceso jurídico de la "apelación", en virtud del cual un ciudadano ateniense, caso de no haber obtenido un resultado satisfactorio ante alguno de los tribunales de los demos, podía recurrir a mayores instancias legales en un intento de alcanzar un fallo, en su opinión, más justo. Pues bien, una simple ojeada al artículo de $\dot{\varepsilon} \varphi$ in $\mu$ en el L-S-J (verbo, como es sabido, de donde deriva el sustantivo é $\varphi \varepsilon \sigma ı)$ ) nos hace ver cómo, en medio de un amplio abanico de valores pertenecientes en su mayoria a la lengua común con el significado general de «enviar» en sus diferentes acepciones contextuales, aparece un incipiente empleo jurídico. Pero si observamos a continuación el material analizado en mento el claro predominio que ha adquirido el valor jurídico frente a los usos comunes paralelos a los encontrados en el verbo: hay un abandono evidente de los sentidos que podríamos calificar de materiales o concretos, como "envio", "lanzamiento", en favor de los significados abstractos, entre los que goza de una relevancia especial su uso en la esfera jurídica con el valor ya mencionado de «apelación».

Es evidente que se podrá alegar el carácter selectivo del material ofrecido por el diccionario inglés. Pero, a mi juicio, hay otro hecho más decisivo.

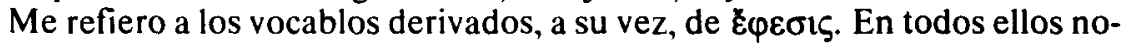
tamos ya un empleo casi exclusivo del sentido legal, en cuyo caso ya no se trata de una mayor o menor intensidad de un valor determinado, sino de una reducción de los significados tradicionales de la lengua común, lo que, en mi opinión, habla a las claras del uso preferente que está adquiriendo el

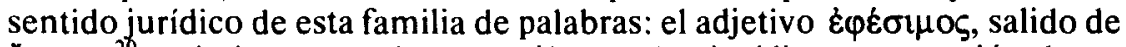
$\varepsilon \varphi \varepsilon \sigma ı \varsigma^{26}$, prácticamente tiene ya sólo empleo jurídico, a excepción de un pasaje en Josefo $(A J 19.250)$ :

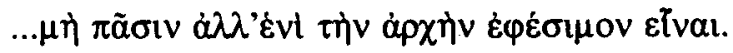

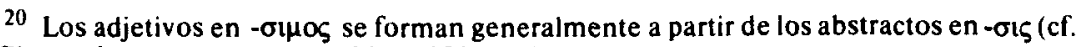
P. Chantraine, op. cit. en nota 16, p. 154 y ss.). 
3.1.3. Uso especializado. Dentro aún del primer gran apartado de términos de la lengua común que, en un momento dado, son utilizados para expresar realidades jurídicas, y dando un paso más en este proceso que voy pergeñando de cómo hay una tendencia clara a un empleo creciente del valor técnico, creo que se puede establecer un tercer apartado, en el que se agruparían una serie de vocablos en los que observamos no ya un uso prevalente en el sentido descrito en el tipo anterior, sino que aqui asistimos a una especialización total con el abandono consiguiente de los diversos valores de la lengua común previos a la aparición del sentido jurídico. Sería, pues, el escalón último de la gradación: la radicalización total a favor del empleo como tecnicismo legal ${ }^{21}$.

Veamos algún ejemplo que nos ilustre tales consideraciones. El término ouvíropos es un claro compuesto formado por el preverbio ouv- y una for-

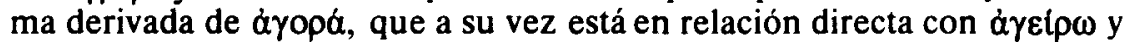

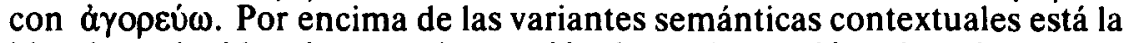
idea de "coincidencia": reunir, reunión, lugar de reunión, plaza del mercado, hablar en reunión, hablar, etc. Pues bien, en esta situación es fácilmente comprensible que un término como ouvíropos, cuyo valor general de "coincidencia» se ve reforzado por la adición del preverbio ouv-, equivalga a nuestro "coincidente», "que está de acuerdo con" en ese pasaje de S. Tr. 1165 en que Heracles hace saber a su hijo que los oráculos nuevos confirman los antiguos:

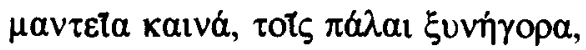

Y algo semejante encontramos en A. Ag. 831, cuando el héroe soberano de Micenas, a su llegada de vuelta del cerco de Troya, expresa su coincidencia de criterio con el pensamiento del coro en torno a la hipocresía humana, para lo que utiliza una expresión tautológica, típica en las manifestaciones de asentimiento 22 :

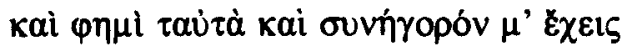

En ambos pasajes ${ }^{23}$ asistimos a un uso aún de lengua común, donde pri-

${ }^{21}$ El calificativo de "total» es, por supuesto, relativo, puesto que se basa en el material selectivo de L-S-J. Pero aunque en la realidad no lo sea, lo que sigue siendo cierto es que se trata de un material mucho más radicalizado que el visto en los apartados anteriores. Por esta razón lo he denominado "uso especializado" y no "uso absoluto", ya que lo que es del todo evidente es su empleo plenamente volcado hacia el valor juridico.

${ }_{22}$ Cf. el comentario de E. Fränkel al Agamenón de Esquilo, Oxford, 1950. Vol. II, p. 384.

23 Mantengo el orden expositivo de L-S-J, que altera la catalogación cronológica en beneficio de criterios sintácticos. 
ma todavía el valor de "coincidencia» entre dos partes. Pero en un momento dado, cuando surge la necesidad de un término específico para la figura jurídica del abogado que "colabora" con una de las partes en la reclamación legal ante los tribunales, el griego antiguo echa mano de este vocablo. Y si es fácilmente perceptible el paso semántico producido, no lo es menos el matiz diferenciador entre uno y otro estadio del proceso, aparte, por supuesto, el hecho de que se constituya como tecnicismo jurídico. Pues bien, una vez alcanzado el cambio semántico podemos observar que nuestro término en cuestión queda radicalizado en su uso para el campo legal, con abandono total de otros posibles contextos paralelos a los ejemplos con que abríamos el análisis.

3.2. Neologismos exclusivamente juridicos. Frente al tipo de vocabulario analizado en el punto anterior, en el que hemos asistido a una progresiva evolución semántica a partir de los valores comunes del léxico general de la lengua, podemos también observar la tendencia constante a ir creando una terminología nueva para satisfacer las necesidades de expresión que van apareciendo en las relaciones legales. Y este procedimiento de los neologismos es el segundo gran filón para la consolidación de un vocabulario jurídico en Grecia. Constatamos, pues, la paulatina aparición de una serie de vocablos con significación originaria y exclusivamente ${ }^{24}$ específica del campo del Derecho.

3.2.1. En un primer nivel lingüistico puede comprobarse que en este área del léxico se mantienen los mismos procedimientos de formación que en el resto del vocabulario griego. Encontramos constantes ejemplos de derivación tradicional: a partir del sustantivo $\psi \tilde{\eta} \varphi \circ$ (término $^{\circ}$ perteneciente originariamente a la lengua común, "canto rodado", pero que en un momento dado adopta también el significado de "voto", dado el procedimiento material que se consolida en Grecia al respecto) surge un verbo con el sufijo - $i \zeta \omega$, tan corriente en la derivación verbal dentro de la tendencia creciente a la gramaticalización de un sistema verbal en griego: $\psi \eta \varphi i \zeta \omega^{25}$, con el valor básicamente ${ }^{26}$ de "votar»; y sobre el término verbal se forma un derivado nominal igualmente convencional por medio del sufijo corriente $-\mu \alpha: \psi \eta \dot{\eta} \varphi \iota \sigma \mu \alpha$, de uso exclusivamente jurídico ${ }^{27}$. También es

24 Este uso exclusivo no se contradice con la posible aparición de un empleo no jurídico, sino de la lengua común, en una época muy posterior y con unafrecuencia de uso reducida a algún caso individual.

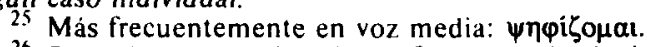

26 Sobre los usos no juridicos, cf. el apartado siguiente de «Neologismos jurídicos con valores posteriores de la lengua común".

${ }_{27}$ L-S-J recoge algunos ejemplos, en los que puede percibirse un cierto empleo metafórico, pero en los que sigue vigente y claro el valor originario de la esfera legal: «los decretos de los dioses». 
normal el proceso inverso, a partir de un verbo: sobre $\varepsilon i \sigma \varphi \varepsilon ́ p \omega$, que junto a valores de la lengua común encontramos el significado técnico de «tribu-

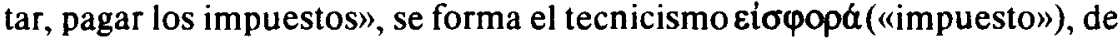
acuerdo con las normas tradicionales de la derivación en griego.

Y lo mismo hay que decir de la composición. Es de todos conocido que, frente al uso restringido de este procedimiento en la lengua común, las diversas terminologías técnicas en griego hicieron de él un empleo creciente, y la parcela jurídica no es una excepción a esa regla. Y ello no sólo en la variante enormemente productiva de un primer término invariable, donde el empleo de las diversas preposiciones como preverbios aporta unas enormes posibilidades expresivas, sino también en los compuestos con ambos térmi-

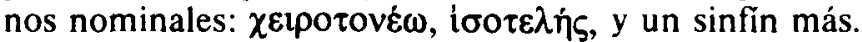

$Y$ finalmente, en tercer lugar, está el campo de los préstamos de otras lenguas. En el caso de Grecia la situación en este terreno es un tanto peculiar. Para empezar, no hay que olvidar la posibilidad, aunque restringida y siempre problemática, de préstamos de lenguas asiáticas ${ }^{28}$. Pero la fuente principal de este tipo de incremento lexical le viene al griego de su contacto directo y estrecho, en un momento dado de su historia, con el latín. Ya a principios de siglo, Magie ${ }^{29}$ estudió esta relación entre una y otra lengua, y llegó a establecer una triple posibilidad de comportamiento en los préstamos del latín al griego: transcripción-calco semántico-ampliación del sentido de un término preexistente, lo que podemos ejemplificar con

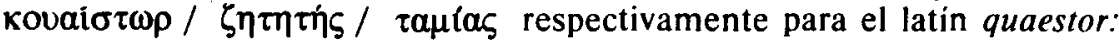

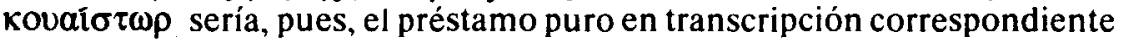

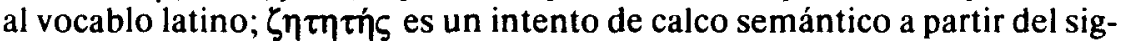

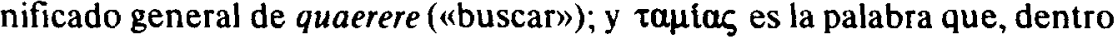
de su amplio campo semántico, abarca de alguna forma el concepto expresado por el término latino ${ }^{30}$. Ahora bien, a este aluvión de términos latinos traspasados al griego hay que hacerle dos precisiones: 1) normalmente son de fecha tardía, puesto que el fenómeno se produce cuando se estrechan las relaciones institucionales entre el mundo griego y el romano, y esto tiene rugar en su mayor parte en época imperial, cuando en suelo egipcio la población griega allí asentada entra en contacto directo con una masa cada vez mayor de colonos romanos; y 2) estos nuevos términos griegos de vocabulario legal-institucional con frecuencia no son de utilidad para el conocimien-

${ }^{28}$ Este es un terreno enormemente delicado. El último trabajo que conozco por estos

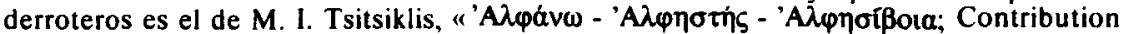
à l'étude de la préhistoire du Droit grec des contrats", Hellenica, 31, 1979, 3-43 (en griego, con resumen en francés, p. 281), que ve en la raiz $\alpha \lambda \varphi$ - un préstamo del fenicio aleph $=$ «(cabeza de) buey"s.

29 D. Magie, De Romanorum iuris publicique sermonis vocabulis sollemnibus in Graecum sermonem conversis, Diss. Leipzig, 1906.

30 Ultimamente tenemos el trabajo de H. Mason, Greek terms for Roman institutions. A lexicon and analysis, Toronto, 1974, sobre la adecuación del griego de los términos institucionales latinos. 
to del Derecho griego, puesto que recubren realidades correspondientes al Derecho romano.

3.2.2. Dentro de este apartado general de la tendencia creciente a la formación de neologismos jurídicos, creo que podemos establecer aún un segundo nivel, prueba todavía más clara a mi juicio de esa corriente consciente en Grecia a ir constituyendo un léxico especializado para el campo del Derecho. Me estoy refiriendo a la creación de neologismos sobre otros neologismos. Y con este tipo de léxico no aludo a procesos normales de derivación, según los cuales se forma un neologismo nominal a partir de otro previo verbal, o viceversa, como hemos visto que sucedía con $\psi \eta \varphi i \zeta \omega$ у $\psi \eta \dot{\varphi} \iota \sigma \mu \alpha$. Ahora estoy pensando más bien en nuevas categorías legales, ante cuya paulatina aparición la lengua intenta crear los términos adecuados, para lo cual se sirve de neologismos preexistentes, sobre los que operará con los procedimientos normales, principalmente de la composición; así, el resultado no es la elaboración de un término nuevo en otra clase gramatical, sino la aparición del vocablo oportuno para la expresión de una realidad jurídica distinta.

Veamos algunos ejemplos. Es de todos conocido el término $\chi \varepsilon \varphi p o \tau o-$ véw («votar a mano alzada»), como variante legal frente a la también posible "votación secreta» $(\psi \eta \varphi$ í $\zeta o \mu \alpha)$, pues bien, en un momento dado de la legislación pública ateniense se introduce la categoría de la "ratificación»" de diversos magistrados en sus cargos al cabo de un tiempo determinado, para lo cual se instruye el procedimiento de una nueva votación, que ahora no será «electiva» sino «ratificadora», y para ello la lengua griega recrea un neologismo: $\dot{\varepsilon} \pi \chi \chi \varepsilon \varphi \tau_{0}=v \varepsilon \omega$, cuya formación lingüística es tradicional, pero cuyo significado es absolutamente técnico del área juridica, sin empleos en ningún otro tipo de contexto, lo que deja ver a las claras que el nuevo vocablo se creó específicamente como tecnicismo legal ${ }^{31}$. Y frente a este empleo del preverbio $\dot{\varepsilon} \pi$ - tal vez sea ilustrativo presentar otro ejemplo que nos ponga de manifiesto la variada funcionalidad y riquezs expresiva del vocabulario griego: en la reunión de la Asamblea, cuando llega el momento de que todos los asistentes «voten» la propuesta previamente presentada, se utiliza el término $\psi \eta \varphi i \zeta o \mu a{ }^{32}$; pero para el acto de que el presidente de la sesión "ponga a votación» la moción en cuestión, el griego dispone de una

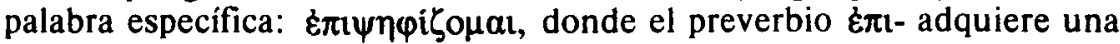
connotación distinta.

31 No obstante, encontramos huellas de vacilaciones, lo que habla del estado embrionario aún de estas terminologias técnicas dentro del léxico griego. Pero sobre este punto volveré después con más detenimiento.

32 Sobre sus posibles empleos positivos ("votar en secreto») o neutro ("votar») hablaré más abajo. 
3.3. Neologismos juridicos con valores posteriores de la lengua común. En este tercer gran apartado se agrupa toda una serie de vocablos que, aunque surgidos históricamente como tecnicismos del campo del Derecho, posteriormente, por los diversos caminos de la evolución semántica, adoptan valores no legales en contextos de la lengua común.

Es, pues, el caso contrapuesto al primer grupo, en el que se partía de la lengua común para especializarse progresivamente en una aceptación jurídica. De otra parte, este tercer tipo no deja de ser realmente una subdivisión del segundo, puesto que en uno y otro se trata de la entrada de neologismos; pero hay una diferencia importante que apoya esta escisión: ahora nos encontramos con que esta serie de nuevos vocablos son adoptados por la lengua común, aunque su intensidad de empleo se mantiene en unos límites siempre reducidos.

Veamos algunos ejemplos. En el articulo correspondiente al verbo $\psi \eta \varphi i \zeta \omega$ en el L-S-J podemos observar cómo, a parte del significado central de "votar", en un momento posterior es utilizado con el valor de "contar" (Plb. V 26.13, AP 11.168, 171, Plu. 2.141C), o con el de "sumar" (Theol. Ar. 64), frente a lo cual se encuentra el empleo primordial y originario de tecnicismo legal para la votación, en este caso de forma secreta mediante el depósito de los $\psi \tilde{n} \varphi 0$ correspondientes. Otro ejemplo: es bien sabido que entre los primeros pasos del mecanismo procesal ateniense estaba la presenta-

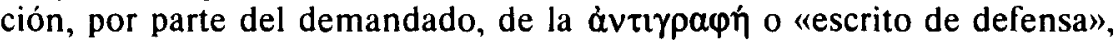
donde intentaba rechazar los cargos alegados por el demandante. Pues bien, este término con el tiempo adoptó también otros valores: «réplica» (como el famoso Anticatón de César en contestación al Catón de Cicerón en Plu. Caes. 3; cf. también Plu. Sol. 1, 2.1059B, Herm. in Phdr 189); "acción de transcribir, copiar" un escrito (D.H. 4.62.6); "rescripto imperial» (OGI 262.27); pero, una vez más, paralelamente a estos valores secundarios se mantiene el empleo central de término jurídico.

La enumeración podría continuarse en páginas sucesivas, pero el cotejo de cualquier obra lexicográfica general nos ahorra el tener que continuar con más ejemplos. Ahora bien, sí es importante hacer algunas reflexiones ante este estado de cosas. En primer lugar, es necesario que los diccionarios recojan con precisión esta realidad léxica, cosa que no suele suceder ni siquiera en el L-S-J, cuyo comportamiento al respecto es variable. Me explico. Si, al menos por la información de que disponemos por la transmisión textual, el valor jurídico es el primero cronológicamente y el más destacado en intensidad de empleo, deberemos deducir que se trata de un neologismo jurídico y, consiguientemente, asignarle el lugar inicial correspondiente a la hora de redactar el artículo lexicográfico pertinente. Y ello no sólo por un prurito cronológico, sino porque nos ofrece la posibilidad de entresacar el correcto camino que siguió en cada caso el proceso de evolución semántica.

Es evidente que, dada la naturaleza del material para el estudio del griego antiguo, siempre es posible que nuestras hipótesis de hoy dia no fueran correctas de haber dispuesto de un estado de cosas semejante al que se ha- 
llan las lenguas modernas. Pero en nuestro caso concreto los datos hablan en el sentido que venimos exponiendo. Y tal vez haya algún apoyo más: nuevamente volvemos a encontrar que el campo de los derivados apoya la interpretación propuesta, puesto que los términos surgidos ahora a partir del neologismo se circunscriben en su mayoria al área semántica jurídica, mientras que son escasos los que adoptan también alguno de los usos derivados de la lengua común.

3.4. Fórmulas juridicas. El mismo proceso de paulatina consolidación de una terminología jurídica en Grecia se puede detectar a nivel de fraseología. Y ello a un doble nivel, en paralelismo a los dos grupos generales de léxico de que he venido hablando: vocabulario de la lengua común especializado con valor jurídico frente a verdaderos neologismos técnicos.

En primer lugar, pues, es plenamente perceptible que una serie de expresiones, que en un primer momento pudieron perfectamente aparecer en contextos indistintos, con el tiempo se fueron restringiendo al área de las relaciones legales, terminando por anquilosarse en verdaderos clichés de la

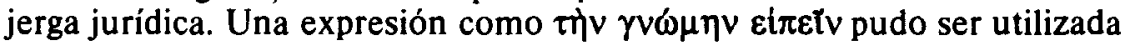
sin una connotación precisa en cualquier tipo de texto, pero no es menos cierto que termina por consagrarse como casi una fórmula fija para el procedimiento legal de ««presentar una moción» en la Asamblea. Otro ejemplo, más esclarecedor por lo que luego diré, es el de la frase tís áyopev́elv

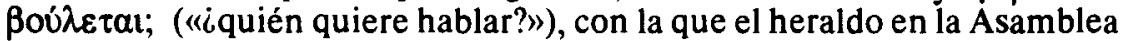
ofrecía la posibilidad de intervenir a cualquiera de los asistentes; y que esta frase convencional terminó por restringirse en gran medida al contexto legal correspondiente nos lo demuestra Aristófanes cuando ironiza al principio de sus Acarnienses sobre el funcionamiento de una sesión de la Asamblea $^{33}$.

Pero también hubo neologismos a este nivel, lo que en cierta medida equivaldría a lo que ahora llamamos lexías complejas, es decir, una expre-

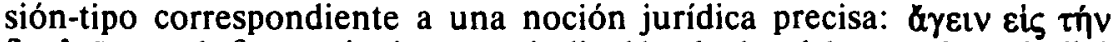

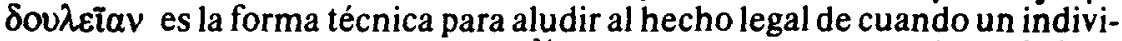

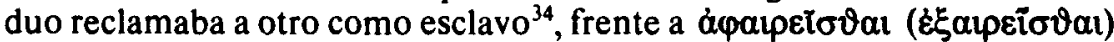

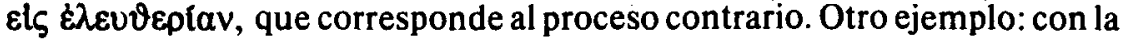

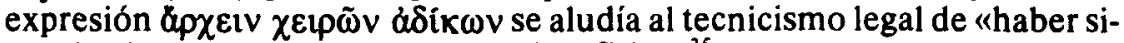
do el primero en iniciar una agresión física» ${ }^{35}$.

33 Aristófanes, Los acarnienses, 45.

34 Este ejemplo, a pesar de su importancia y notoriedad, es una prueba evidente del descuido con que en ocasiones se trata en L-S-J el vocabulario jurídico.

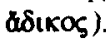

35 Nuevo ejemplo del desafortunado tratamiento lexicográfico en L-S-J (cf. s. $u$. 


\section{EVOLUCIÓN SEMÁNTICA}

Como el restante vocabulario, también esta parcela de la terminología jurídica experimenta una serie de alteraciones semánticas de indole diversa, según se trate de factores internos o externos a la lengua.

4.1. Evolución intraverbal. También este vocabulario se ve afectado por las mismas presiones intralingüisticas de estructuración y reestructuración que el resto del léxico general. Y así, una primera consideración podría ser la de observar la tendencia a crear usos neutros dentro de una oposición previa con ambos términos positivos: $\delta \tilde{\eta} \mu$ o $\zeta$ es «el pueblo como

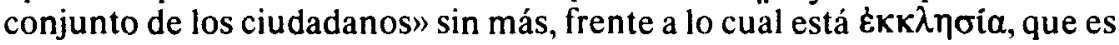
«el pueblo reunido en la Asamblea»; pues bien, en un momento dado comenzamos a ver empleado el primer término de la oposición con el valor especializado de «la Asamblea». Algo semejante se produce en la oposición

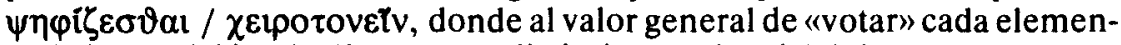
to de la oposición añadía un rasgo distintivo en virtud del sistema concreto a seguir: votación secreta (mediante la introducción de los $\psi \tilde{\eta} \varphi 01$ en las urnas correspondientes) frente a votación abierta a mano alzada, estando, además, adscrito cada procedimiento a una institución diferente: el primero al tribunal de justicia, el segundo a la Asamblea; sin embargo, encontramos varios ejemplos de $\psi \eta \varphi i \zeta \zeta \varepsilon \sigma \vartheta \alpha$ como sinónimo de $\chi \varepsilon i \rho o \tau o v \varepsilon t v$ en el procedimiento de la cámara popular, lo cual hay que interpretarlo no como una alteración del sistema material de votación, sino como una variación en el tipo de oposición semántica.

Otro reflejo de evolución intraverbal es la tendencia de la lengua a la reestructuración reguladora del sistema de oposiciones. Y en este sentido hay que entender una serie de creaciones paralelas que dan uniformidad al sistema; por ejemplo: al lado del verbo $\chi \varepsilon \varphi p o$ tovetv existe desde época temprana un sustantivo de acción, $\chi \varepsilon \varphi \rho \tau o v l a$, cosa que no sucede con su para-

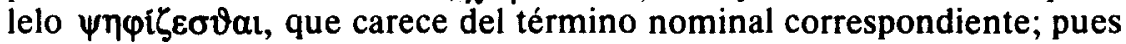
bien, en un momento dado se crea el vocablo $\psi \eta \varphi \circ \varphi o p i \alpha$, que viene a ocupar el hueco existente. E igualmente hay que interpretar de la misma forman los intentos de redistribución de las series de sinónimos: términos

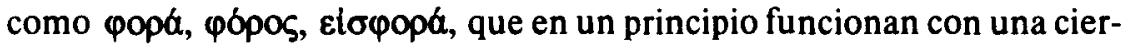
ta indiferencia semántica, experimentan una presión sistematizadora, que los aboca a que popá permanezca como término genérico, mientras que pópos tiende a radicalizarse en la acepción de «tributo impuesto a otras

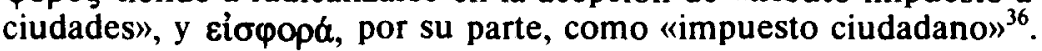

36 Observemos que, de acuerdo con las categorias arriba establecidas, $\varphi$ opa pertenecería al tipo "vocabulario común con valor jurídico en uso alternativo", $\varphi$ óps al de "vocabulario común con valor jurídico en uso preferente», y ei $\sigma \varphi \circ \alpha$ al de "neologismo". 
4.2. Evolución extraverbal. Dado el tipo de léxico aquí tratado, es del todo evidente que hay una constante alteración del fondo semántico de los términos, en función de la variación a que se ven sometidas históricamente las diversas instituciones legales que aquéllos expresan. $O$ dicho más sencillamente, un mismo vocablo jurídico puede no recubrir la misma realidad legal en dos momentos distintos, dadas las constantes variaciones que la sociedad va introduciendo en su funcionamiento.

\section{USOS IMPRECISOS}

Hasta aquí he venido esforzándome en destacar las diversas circunstancias que apoyan la hipótesis de la existencia de un vocabulario jurídico en Grecia. Ahora bien, no es menos cierto que nos encontramos aún en un estadio inicial, en el que son perceptibles ciertos titubeos. Y querría ahora aludir a algunos de ellos, para que la visión global del panorama descrito sea más exacta.

Lo característico de un léxico técnico bien consolidado es que cada término sea univoco y claramente deslindado de otras parcelas semánticas, puesto que ha sido introducido para reproducir una realidad muy concreta, al menos sincrónicamente. Sin embargo, en el caso del griego nos encontramos con algunos vocablos que, aún dentro del campo específicamente jurídico y sólo dentro de él, se emplean para varios significados legales. Pongamos

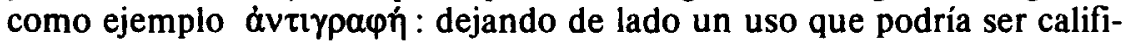
cado de no sincrónico con los restantes ya que sólo lo encontramos atestiguado a finales del siglo $\mathrm{V}^{37}$, puede comprobarse que en el mismo Demóstenes aparece con tres sentidos distintos: 1) como la "defensa» que el demandado debe presentar ante el tribunal en respuesta a las reclamaciones del demandante (D. XLV, 1);2) como "recriminación», o sea, cuando el acusado en su defensa responde a su vez con otra acusación contra el teóricamente dañado (D. XLII, 17); y 3) como «reivindicación o recurso», en el caso de que, una vez asignada una herencia, algún individuo se sienta lesionado y reivindique su derecho (D. XLIII, 16). Ante tal estado de cosas es fácil llegar a la conclusión de que el griego se encuentra a medio camino del recorrido: ha creado un término técnico para todo tipo de respuesta por escrito ante cualquier acción legal, pero no ha llegado aún a matizar las posibles variantes existentes.

Otra clase de imprecisión afecta a la creación de ese grupo de neologismos formados a su vez sobre otros neologismos. Lo esperable, desde la perspectiva de nuestras lenguas modernas, es que ese hiperneologismo tenga un significado muy concreto y específico, dado el proceso de su forma-

${ }^{37}$ Se trata de su empleo como sinónimo de raparpaph en Lisias 23.5,10. Ya este mismo hecho de la sinonimia no deja de ser otro reflejo de las imprecisiones a que en este momento me estoy refiriendo. 
ción, dentro ya de un área muy concreta. Sin embargo, en el caso del griego observamos situaciones de fluctuación en las que el nuevo término es simplemente un sinónimo del vocablo que le ha servido de punto de partida: un ejemplo muy claro lo tenemos en La constitución de los atenienses de Aristó-

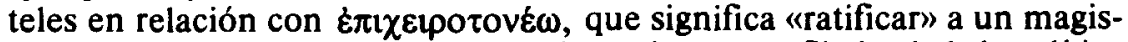
trado en el cargo que ya ocupaba desde un tiempo prefijado, dada la política ateniense de la época de revisar periódicamente la actuación de sus diversos funcionarios públicos; $y$ esto es precisamente lo que encontramos en el párrafo 43.4:

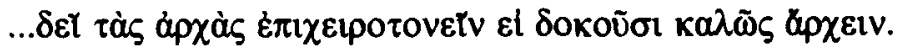

«ratificar las magistraturas si se estima que las están desempeñando oportunamente». Pero unas líneas más abajo, 43.5 , nos encontramos con un uso neutro del mismo término, donde vemos que equivale al neologismo que le sirvió de base, $\chi \varepsilon \iota \rho \circ \tau o v \varepsilon \omega$ :

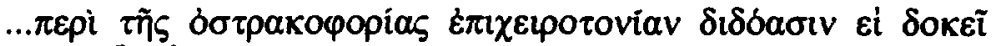

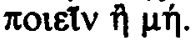

"conceden una votación a mano alzada sobre el ostracismo, si se estima oportuno proceder a él o no", donde es imposible interpretar el término en el sentido técnico arriba aludido, valor éste, por supuesto, que volvemos a encontrar en otros pasajes.

A estos y otros hechos de tipo lingüístico vienen a sumarse realidades extralingüísticas del propio campo de las relaciones juridicas, que en el caso de Grecia, y como se sabe, no llegaron a constituir un corpus armónico y cerrado. Y esta realidad se refleja lógicamente en el vocabulario, en el que, por ejemplo, vemos que hay en ocasiones términos diferentes según se trata de un hombre o de una mujer: en paralelismo a la conocida dualidad de $\gamma \alpha \mu \varepsilon \omega$

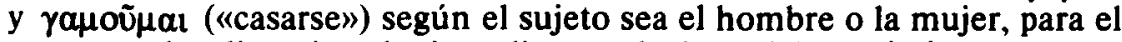
concepto de «divorcio» el griego dispone de dos palabras: ḋ $\pi \delta \varepsilon \mu \psi(\varsigma$, por parte del marido, lo que podría traducirse por algo así como "devolución»

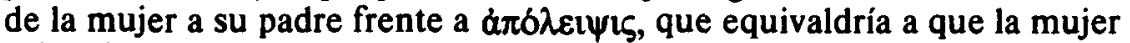
"abandona» a su esposo.

En definitiva, son perfectamente detectables toda una serie de vacilaciones en el vocabulario jurídico griego, reflejo de su estado incipiente de formación y de la falta de un ordenamiento riguroso de las propias relaciones legales en el Derecho griego. Pero esto no va en contra de que estemos asistiendo al proceso de su constitución, y de que, como vehículo de las diversas categorías legales, se hayan ido o bien radicalizando en un uso jurídico todo un grupo de vocablos de la lengua común, o bien creando una serie de neologismos especificos para esta área del léxico. 